ПРИМЕНЕНИЕ НАВИГАЦИОННОЙ РИТМИЧЕСКОЙ ТРАНСКРАНИАЛЬНОЙ МАГНИТНОЙ СТИМУЛЯЦИИ С ЦЕЛЬЮ КОРРЕКЦИИ ПИЩЕВОГО ПОВЕДЕНИЯ ПРИ ОЖИРЕНИИ (КЛИНИЧЕСКИЕ НАБЛЮДЕНИЯ)

() П.И. Кузнецова ${ }^{1 *}$, О.В. Логвинова ${ }^{2}$, А.Г. Пойдашева', Е.И. Кремнева' ${ }^{1}$, И.С. Бакулин ${ }^{1}$, А.А. Раскуражев ${ }^{1}$, О.В. Лагода', Н.А. Супонева', Е.А.Трошина², М.М. Танашян ${ }^{1}$, М.А.Пирадов ${ }^{1}$

'Научный центр неврологии, Москва, Россия;

${ }^{2}$ Национальный медицинский исследовательский центр эндокринологии, Москва, Россия

Ожирение представляет собой патологическое состояние, вызванное избыточной массой тела и требующее медицинского вмешательства. Накопленный за прошедшие десятилетия клинический и научный опыт позволяет рассматривать эту проблему как самостоятельное заболевание со своими собственными патофизиологическими особенностями, распространенностью, заболеваемостью, подходами к терапии и профилактике. Одним из важнейших факторов патогенеза ожирения является нарушение пищевого поведения, центральная регуляция которого осуществляется при активном участии префронтальной коры головного мозга. Воздействие на эту область (например, с помощью неинвазивной стимуляции головного мозга) может явиться одним из перспективных способов модулирования пищевого поведения. В настоящей статье представлен собственный опыт лечения морбидного ожирения с помощью инновационного метода навигационной ритмической транскраниальной магнитной стимуляции (рТМС). Проиллюстрированы изменения активации дорсолатеральной префронтальной коры (ДЛПФК) по данным функциональной магнитно-резонансной томографии до и после курса рТМС. Рассмотрены также возможные механизмы влияния ДЛПФК на формирование пищевого поведения. Данные клинические наблюдения подтверждают важную роль ДЛПФК в развитии нарушений пищевого поведения, а также демонстрируют потенциальную эффективность нейромодуляции в коррекции такого рода расстройств.

КЛЮЧЕВЫЕ СЛОВА: ожирение; пищевое поведение; транскраниальная магнитная стимуляция; функциональная магнитно-резонансная томография; дорсолатеральная префронтальная кора; клинический случай.

\title{
NAVIGATED REPETITIVE TRANSCRANIAL MAGNETIC STIMULATION TO CORRECT EATING BEHAVIOR IN OBESITY (CLINICAL CASES)
}

(c) Polina I. Kuznetsova1*, Oksana V. Logvinova², Alexandra G. Poydasheva1', Elena I. Kremneva1, Ilya S. Bakulin',

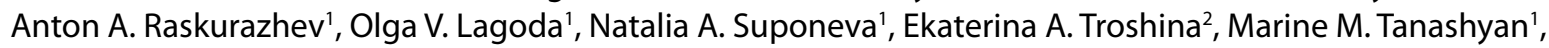
Michael A. Piradov ${ }^{1}$

${ }^{1}$ Research Center of Neurology, Moscow, Russia;

${ }^{2}$ Endocrinilogy Research Centre, Moscow, Russia

Obesity is a pathological condition caused by overweight and requiring medical intervention. The clinical and scientific experience gained over the past decades has allowed researchers to consider this problem as an independent disease with its own pathophysiological features, prevalence, incidence, approaches to therapy and prevention. One of the most important factors in the pathogenesis of obesity is disordered eating behavior, the central regulation of which is carried out with the active participation of the prefrontal cortex. Impact on this area (for example, using non-invasive brain stimulation) may be one of the promising ways to modulate eating behavior. The article describes clinical cases of treatment of morbid obesity using navigated rhythmic transcranial magnetic stimulation (rTMS). Different patterns of dorsolateral prefrontal cortex (DLPFC) activation before and after rTMS are demonstrated. Possible mechanisms of the influence of DLPFC on the formation of eating behavior are also considered. These data underline the important role of DLPFC dysregulation in obesity, as well as show potentially effective neuromodulation techniques.

KEYWORDS: obesity; eating behavior; functional transcranial magnetic stimulation; dorsolateral prefrontal cortex; case report.

\section{АКТУАЛЬНОСТЬ}

Распространение ожирения на сегодняшний день приняло характер пандемии. С 1975 по 2016 гг. число людей, страдающих ожирением, во всем мире выросло более чем втрое [1]. В 2013 г. Американская медицин- ская ассоциация признала ожирение заболеванием [2], ее примеру последовали ряд других медицинских организаций, включая Всемирную организацию здравоохранения [3]. В 2015 г. Нагойская декларация определила «ожирение» как патологическое состояние, вызванное избыточной массой тела и требующее медицинского 
вмешательства. В настоящий момент различные ассоциации врачей во всем мире рассматривают эту проблему как самостоятельное заболевание со своими собственными патофизиологическими особенностями, распространенностью, заболеваемостью, подходами к терапии и профилактике $[4,5]$.

Ожирение значительно увеличивает расходы государства на здравоохранение - как за счет лечения собственно ожирения, так и ассоциированных с ним осложнений, к которым относятся сердечно-сосудистые заболевания [6], сахарный диабет 2 типа, нарушения липидного и пуринового обменов, желчнокаменная болезнь, заболевания суставов, синдром обструктивного апноэ сна и прочие [7].

Помимо социальных и экономических потерь в обществе в результате пандемии ожирения, необходимо отметить стигматизацию пациентов с данной патологией и развитие аффективных расстройств на этом фоне [8].

В основе терапии избыточной массы тела и ожирения любой степени лежат прежде всего коррекция питания пациента, включающая в себя постепенное снижение калорийности суточного рациона, рациональное распределение калоража в течение дня, достижение оптимального соотношения макро- и микронутриентов с учетом имеющихся осложнений ожирения и сопутствующих заболеваний, а также коррекция физической активности пациента с целью увеличения объема энерготрат. В то же время рациональное питание и достаточная физическая активность сами по себе являются факторами, лежащими в основе профилактики развития ожирения, и несмотря на то, что данные факторы являются модифицируемыми (воздействие на них может и предотвратить, и лечить уже имеющееся ожирение), пациенты сталкиваются с рядом трудностей на пути изменения сложившегося годами стереотипа питания и гиподинамии. Большой вклад в низкую и/или краткосрочную эффективность диетотерапии вносит имеющееся у пациента нарушение пищевого поведения (ПП), основными типами которого являются экстернальный, эмоциогенный и ограничительный (часто встречается их комбинация) $[9,10]$. Кроме нарушений пищевого поведения, к вероятным причинам, способствующим развитию ожирения, относят генетическую предрасположенность, нарушения в работе оси «кишечник-головной мозг», патологические изменения регуляции секреции инкретинов и антиинкретинов, количественное и качественное изменение состава микробиоты кишечника, повреждения дофаминергических и серотонинергических сигнальных путей в головном мозге и еще целый ряд факторов [11-13]. Для достижения клинически значимого и долговременного снижения массы тела, а также облегчения формирования у пациента новых принципов питания возможно использование фармакотерапии ожирения. Основными требованиями к данным препаратам является не только их доказанная эффективность, но и сердечно-сосудистая безопасность, а также отсутствие привыкания и минимизация возможных побочных эффектов. Ни один из имеющихся на текущий момент препаратов для лечения ожирения не одобрен для пожизненного приема [14-16]. В этой связи в ряде случаев после прекращения приема фармакотерапии может иметь место постепенный рикошетный набор массы тела. Кроме того, не более $10 \%$ па- циентов с морбидным ожирением достигают целей лечения, несмотря на применение всех вышеуказанных методов, поэтому в данной группе может быть рекомендовано хирургическое лечение. Проведение бариатрических операций возможно при морбидном ожирении и неэффективности ранее проводимых консервативных мероприятий у лиц с ИМТ $\geq 40$ кг/м² (независимо от наличия сопутствующих заболеваний) и при ИМТ $\geq 35 \mathrm{kг} / \mathrm{M}^{2}$ при наличии сопутствующих заболеваний, на течение которых можно воздействовать путем снижения массы тела. Однако хирургическое лечение ожирения имеет целый ряд абсолютных и относительных противопоказаний, а некоторые варианты операций требуют последующего пожизненного приема витаминов и микроэлементов. Важным аспектом является командное решение о проведении хирургического лечения ожирения с участием психиатра, поскольку при выраженных расстройствах пищевого поведения данный метод также не гарантирует удержания достигнутого результата [17-19].

Как было упомянуто выше, одним из важнейших факторов патогенеза ожирения является нарушение пищевого поведения, центральная регуляция которого осуществляется при активном участии префронтальной коры головного мозга. Воздействие на эту область (например, с помощью неинвазивной стимуляции головного мозга) может явиться одним из перспективных способов модулирования пищевого поведения. К технологиям неинвазивной стимуляции головного мозга относятся две методики: транскраниальная магнитная стимуляция (TMC) и транскраниальная электрическая стимуляция (ТЭС). По данным зарубежных исследований, при сравнении эффектов ТМС и ТЭС на пищевое поведение более высокую эффективность продемонстрировала ТМС [20].

Ритмическая ТМС - метод неинвазивной стимуляции головного мозга, основанный на принципе электромагнитной индукции и состоящий в последовательном нанесении нескольких (как правило, более 1000) стимулов с заданной постоянной частотой. Показано, что рТМС оказывает долговременные эффекты на возбудимость коры, обусловленные влиянием на механизмы синаптической пластичности: долговременное потенцирование (LTP - om англ. long-term potentiation) и долговременное ингибирование (LTD - om англ. long-term depression), в свою очередь опосредованные модуляцией глутаматных NMDA-рецепторов (N-метил-D-аспартат) постсинаптической мембраны [21, 22]. Кроме того, обсуждаются эффекты рТМС на секрецию нейротрансмиттеров и нейротрофических факторов, генетический аппарат нейронов, глиальные клетки, предотвращение клеточной гибели, а также собственные биофизические эффекты магнитного поля [23]. Эффект стимуляции зависит от частоты предъявления стимулов: высокочастотная стимуляция ( $\geq 5$ Гц) вызывает LTP-подобные эффекты, тогда как низкочастотная ( $\leq 1$ Гц) - LTD-подобные [24]. В свою очередь, LTP приводит к усилению синаптической передачи, тогда как LTD, напротив, приводит к длительному ослаблению силы синапсов $[25,26]$. С учетом существующих концепций развития пищевой зависимости одним из основных подходов к применению ТМС является стимуляция активности областей, участвующих в когнитивном контроле, таких как дорсолатеральная префронтальная кора (ДЛПФК) [27]. Для этого наиболее часто использу- 
ется высокочастотная рТМС левой или правой ДЛПФК. Предполагается, что изменение возбудимости ДЛПФК может привести к облегчению когнитивного контроля за приемом пищи и подавлению механизмов вознаграждения, которые приводят к избыточному употреблению пищи $[27,28]$. В ряде исследований было показано, что высокочастотная рТМС левой ДЛПФК способна снижать влечение к пище, уменьшать частоту компульсивного поведения, а также вызывать снижение массы тела [29-31].

В последние годы при проведении рТМС используются навигационные системы, обеспечивающие возможность персонифицированного выбора мишени согласно анатомической структуре мозга или его функциональной активности, а также возможность контроля положения индуктора в течение сессии и повторения ее положения в течение курса.

Одним из подходов к поиску мишени для неинвазивной стимуляции мозга является проведение функциональной МРТ, измеряющей гемодинамический ответ (изменение кровотока), связанный с активностью нейронов головного мозга [32]. Перед серией процедур пациенту выполняется функциональная МРТ (фМРТ-картирование), где на основании его индивидуальных пищевых привычек визуализируются зоны повышенной активации в области ДЛПФК доминантного полушария головного мозга, и далее выбирается зона для рТМС. Известно, что префронтальная часть лобной коры, в частности, ее дорсолатеральные отделы, играет ключевую роль в формировании, организации, планировании и анализе поведения. Нарушение регуляции активности в области левой ДЛПФК может вести к нарушению пищевого поведения, увеличению калорийности выбранных пищевых продуктов, снижению самоконтроля и развитию на этом фоне ожирения. Этим обусловлен выбор данной области в качестве цели для проведения рТМС [33-35].

Далее будут представлены 2 клинических случая первого на территории Российской Федерации применения навигационной рТМС с целью коррекции пищевого поведения у пациенток (мать и дочь) с экзогенно-конституциональным ожирением.

Обе пациентки первично обратились в консультативно-диагностический центр (КДЦ) ФГБУ «Национальный медицинский исследовательский центр эндокринологии» Минздрава России. Ведущей жалобой в обоих случаях являлось наличие избыточной массы тела и пристрастие к употреблению сладостей (шоколад и выпечка) во второй половине дня. Учитывая наличие у обеих пациенток морбидного ожирения без осложнений, эмоциогенного типа нарушения пищевого поведения (подтвержденного по данным опросника DEBQ (The Dutch Eating Behaviour Questionnaire)), пристрастия к быстроусваиваемым углеводам, а также сложностей в соблюдении принципов рационального питания без применения вспомогательных методов (то есть нативно, без назначения, например, фармакотерапии ожирения и пищевого поведения, курса когнитивно-поведенческой психотерапии и т.п.), пациенткам было предложено участие в научном исследовании «Оценка эффективности ритмической транскраниальной магнитной стимуляции (ТMC) при ожирении и метаболическом синдроме», проводимом совместно ФГБУ «Национальный медицинский исследовательский центр эндокринологии» Минздрава Рос- сии и ФГБНУ «Научный центр неврологии». Пациентки не имели противопоказаний со стороны эндокринологической части обследования, в связи с чем были направлены в «Научный центр неврологии».

Исследование было одобрено локальным этическим комитетом «Научного центра неврологии» (№3/15 от 25.02. 2015 г.); обе пациентки подписали письменное информированное согласие на участие в данном исследовании.

\section{ОПИСАНИЕ СЛУЧАЯ 1}

Пациентка Ш., 46 лет (мать): из анамнеза известно, что в детстве динамика изменения массы тела соответствовала полу и возрасту. Минимальная масса тела во взрослом возрасте в 18 лет - 56 кг, максимальная на момент обращения - 113 кг, рост - 164 см, ИМТ 42,0 кг/м² (ожирение 3 степени). За время первой беременности (дочерью, клинический случай которой будет описан ниже) набрала 20 кг, вес дочери при рождении 3900 г, через месяц после родов масса тела матери снизилась на 10 кг. В дальнейшем наблюдалось постепенное медленное увеличение массы тела на 3-4 кг в год, с более выраженным ее нарастанием за последние 3 года. В анамнезе попытки самостоятельно снижения массы тела с помощью уменьшения калорийности суточного рациона (вела примерный подсчет килокалорий по онлайн-калькулятору) с ограничением употребления быстро усваиваемых углеводов и сладостей, однако максимальный срок соблюдения данных принципов питания составлял не более 3 нед, в связи с чем имел место обратный набор массы тела на фоне возвращения к привычному рациону питания. На момент обследования питание включало в себя два основных приема пищи (завтрак и ужин) и 2 перекуса в течение дня на работе (фруктами либо хлебобулочными изделиями). Пациентка подтверждала наличие гиперфагической реакции на стресс, которая проявлялась в «заедании» сниженного настроения в вечернее время (как правило, после ужина или перед сном) сладостями и выпечкой. При анализе дневника питания обращало на себя внимание сниженное количество растительной клетчатки и белка в рационе, с преобладанием быстро усваиваемых углеводов и животных жиров. Физическая активность труда и быта была сниженной. Сопутствующим заболеванием являлся первичный гипотиреоз вследствие хронического аутоиммунного тиреоидита с 2008 г., который на момент обращения в клинику был компенсирован на фоне приема 75 мкг левотироксина в сутки: ТТГ - 1,4 мМЕ/мл (норма 0,31-4,20). Никакой другой медикаментозной терапии пациентка не принимала. Менструации регулярные, беременность в ближайшее время пациентка не планировала. При физикальном осмотре: ожирение по абдоминальному типу с окружностью талии 110 см. На коже живота бледные стрии, которые, со слов пациентки, появились более 10 лет назад. Среднее артериальное давление (АД) при трехкратном измерении было 136/90 мм рт. ст., частота пульса - 60 ударов в минуту, тоны сердца приглушены, ритмичны, патологические шумы не выслушивались. Отеков, выделений из сосков, избыточного оволосения, признаков акромегалии на момент осмотра не выявлялось. Стул регулярный. 
При обследовании в КДЦ получены следующие результаты: в общем анализе крови - без клинически значимых изменений; в биохимическом анализе крови показатели липидного, пуринового, углеводного обмена, уровень креатинина, кальция общего, натрия, печеночных ферментов в пределах референсного диапазона. Уровень гликированного гемоглобина - 5,5\%; уровень пролактина в норме. При ультразвуковом исследовании органов брюшной полости выявлялись эхографические признаки неалкогольной жировой болезни печени. Далее пациентка была направлена в ФГБНУ «Научный центр неврологии» с целью скрининга на предмет выявления противопоказаний к фМРТ и рТМС и, в случае их отсутствия, включения в протокол исследования.

При поступлении в ФГБНУ «Научный центр неврологии» в соматическом статусе: состояние удовлетворительное, положение вынужденное. Кожные покровы и видимые слизистые физиологической окраски, умеренной влажности, чистые. На правой голени визуализируются варикозно расширенные вены. Сердечные тоны приглушены, шумов нет, пульс ритмичный. В легких выслушивается везикулярное дыхание, хрипов нет. чДД 16 в минуту. Живот при пальпации мягкий, безболезненный во всех отделах. Симптом поколачивания отрицательный с обеих сторон. Мочеиспускание безболезненное.

В неврологическом статусе: сознание ясное, менингеальных знаков нет. Черепные нервы интактны. Ограничение движений в шейном отделе позвоночника, подъема правой руки. Парезов нет. Мышечный тонус не изменен. Сухожильные рефлексы живые, D=S. Патологических стопных знаков нет, рефлексы орального автоматизма отсутствуют. Чувствительных нарушений на момент осмотра не выявлено. Координаторные пробы выполняет удовлетворительно. В позе Ромберга слегка пошатывается. Пальпация паравертебральных точек в шейном отделе, надплечье с обеих сторон, краниальных мышц болезненна. Походка не изменена.

\section{ОПИСАНИЕ СЛУЧАЯ 2}

Пациентка Д., 20 лет (дочь): масса тела при рождении - 3900 г, избыточная масса тела с 10-летнего возраста. В возрасте 12 лет обращалась к детскому эндокринологу, были исключены вторичные причины ожирения и рекомендована диетотерапия и интенсификация физической активности, однако в целом данные рекомендации не выполнялись, в связи с чем имело место дальнейшее постепенное увеличение массы тела. На момент обследования питание включало в себя три основных приема пищи (завтрак, обед и ужин), наибольшее количество калорий пациентка употребляла именно в вечернее время. Пациентка также подтверждала наличие гиперфагической реакции на стресс, гиподинамии. Сопутствующие заболевания отрицала, никакой медикаментозной терапии на момент обращения не принимала. Менструации регулярные, беременность в ближайшее время пациентка не планировала. При физикальном осмотре: масса тела - 135,5 кг, рост - 166 см, ИМТ - 49,2, ожирение по абдоминальному типу с окружностью талии 118 см. Среднее артериальное давление (АД) при трехкратном измерении было 122/70 мм рт. ст., частота пульса - 76 ударов в минуту, тоны сердца приглушены, ритмичны, патологические шумы не выслушивались. Отеков, выделений из сосков, избыточного оволосения, признаков акромегалии на момент осмотра не выявлялось. Стул регулярный. При обследовании в КДЦ общий, биохимический и гормональный анализы крови без клинически значимых изменений, уровень гликированного гемоглобина - 4,8\%. При ультразвуковом исследовании органов брюшной полости выявлялись эхографические признаки неалкогольной жировой болезни печени.

После анализа дневников питания обеих пациенток им были даны персонализированные рекомендации по коррекции калорийности и состава рациона питания, а также рекомендована интенсификация физической активности - прохождение не менее 10 тысяч шагов в сутки, а также введение не менее 150 мин дополнительной аэробной физической активности в неделю, разделенных на 3 сессии длительностью по 50 мин с целевой чСС в диапазоне 70-90\% индивидуальной максимальной ЧСС $[36,37]$. Далее пациентка была направлена в ФГБНУ «Научный центр неврологии» с целью скрининга на предмет выявления противопоказаний к фМРТ и рТМС и, в случае их отсутствия, включения в протокол исследования. Учитывая отсутствие противопоказаний, пациентка была госпитализирована в ФГБНУ «Научный центр неврологии» в течение недели.

При поступлении в ФГБНУ «Научный центр неврологии» в соматическом статусе: состояние удовлетворительное, положение вынужденное. Кожные покровы и видимые слизистые физиологической окраски, умеренной влажности, чистые. Периферических отеков нет. Сердечные тоны ясные, шумов нет, пульс ритмичный. В легких выслушивается везикулярное дыхание, хрипов нет. ЧДД 16 в минуту. Живот при пальпации мягкий, безболезненный во всех отделах. Симптом поколачивания отрицательный с обеих сторон. Мочеиспускание безболезненное.

В неврологическом статусе: сознание ясное, контактна, адекватна, ориентирована. Речь не нарушена. Менингеальных знаков нет. Черепные нервы интактны. Ограничение активных и пассивных движений в поясничном отделе позвоночника. Парезов нет. Мышечный тонус не изменен. Сухожильные рефлексы живые, D=S. Патологических стопных знаков нет, рефлексы орального автоматизма отсутствуют. Чувствительных нарушений на момент осмотра не выявлено. Координаторные пробы выполняет удовлетворительно. В позе Ромберга слегка пошатывается. Пальпация паравертебральных точек в нижнегрудном, поясничном отделах болезненна.

\section{Методы исследования и терапевтического}

воздействия, применявшиеся в обоих случаях

Одним из основных противопоказаний для проведения рТМС является наличие пароксизмальных состояний по клиническим данным и/или эпиактивности, пациенткам была проведена электроэнцефалография с целью ее исключения: признаков нарушения биоэлектрической активности мозга и эпилептиформной активности не обнаружено. Пациентки не страдали клаустрофобией, не имели металлических элементов и имплантов. Таким образом, противопоказаний для проведения фМРТ и рТМС выявлено не было. 
Нами был использован протокол, соответствующий имеющимся рекомендациям по безопасному применению рТМС [38]. Описание протокола (указание частоты, интенсивности стимуляции, длительности трейна и интертрейнового интервала, общее количество стимулов в сессии) также соответствует имеющимся рекомендациям и дано таким образом, чтобы оно могло быть повторено независимой группой исследователей. Основным отличием используемого нами подхода было применение фМРТ с оригинальной парадигмой для определения мишени для стимуляции.

Обеим пациенткам проводилась высокочастотная ритмическая нТМС левой ДЛПФК с использованием следующего протокола: частота стимуляции - 20 Гц, интенсивность стимуляции - 100\% моторного порога покоя, длительность пачки стимулов - 2 с, длительность интервала между пачками - 28 с, общее количество стимулов за сессию - 2400. Моторный порог покоя определялся в «горячей точке» как минимальная интенсивности стимуляции, при которой не менее чем 5 стимулов из 10 вызывают формирование вызванного моторного ответа (BMO) с амплитудой более 50 мкВ при условии полного расслабления исследуемой мышцы. Ритмическая нТМС проводилась на аппарате Magstim Rapid 2 (Великобритания), калиброванном под нейронавигационную систему, с использованием восьмиобразной катушки. Курс лечения обеих пациенток включал 18 сеансов, проводимых с понедельника по субботу включительно с перерывом на 1 день (воскресенье) последовательно в течение 3 нед. Важно отметить, что, в отличие от большинства исследований, в которых мишень для стимуляции выбирается стандартно по анатомическим ориентирам, в данном случае использовался персонализированный подход к подбору мишени на основе анализа данных функциональной МРТ (рис. 1, 2). МРТ-исследование проводилось на томографе Siemens MAGNETOM Verio с индукцией магнитного поля 3 Т. Для получения структурной МРТ использовался режим Т1-градиентное эхо (основные параметры режима - время повторения 1900 мс, время

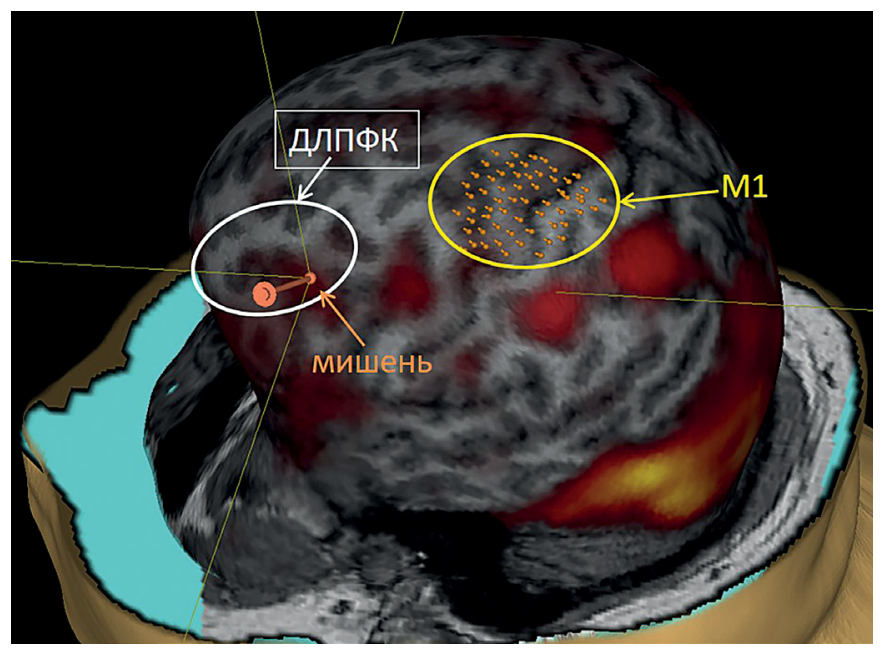

Рисунок 1. Изображение мишени для стимуляции, соответствующей активации в левой ДЛПФК

в нейронавигационной системе (пациентка Д.).

M1 - первичная моторная кора; ДЛПФК - дорсолатеральная префронтальная кора эхо - 2,47 мс, толщина среза - 1 мм, 176 сагиттальных срезов, полностью покрывающих головной мозг). Структурные данные в дальнейшем импортировались в систему навигационной ТМС. Для локализации мишени для стимуляции проводилась функциональная МРТ в режиме Т2-градиентное эхо (время повторения - 3000 мс, время эхо - 30 мс , толщина среза - 3 мм, 36 аксиальных срезов) с применением блоковой зрительной парадигмы собственной разработки, с чередующимся предъявлением нейтральных стимулов (спокойные пейзажи) и высококалорийной пищи (исследование проводилось натощак) (рис. 3, 4).

Результаты оценки динамики зон активации в проекции левой ДЛПФК и клинический эффект

в обоих случаях

Переносимость метода была удовлетворительной. Побочных эффектов со стороны нервной системы (цефалгический синдром, изменение настроения), а также со стороны желудочно-кишечного тракта (тошнота) отмечено не было. Через 3 мес после проведенного лечения: вес матери - 106,0 кг, вес дочери - 122,8 кг. Учитывая уменьшенное потребление калорийной пищи за время госпитализации (последний прием пищи за день - низкокалорийный ужин в 18:00), а также после нее, необходимо принимать во внимание не только эффект от проводимой рТМС, но и приверженность диете. Обе пациентки отмечали снижение выраженности гиперфагии в вечернее время, а также снижение пристрастия к сладкой пище.

По данным фМРТ с пищевой парадигмой, на фоне проведенных сеансов рТМС определяется снижение активации в проекции левой ДЛПФК, которое в рамках настоящего исследования может быть расценено в контексте изменения пищевого поведения. У обследованных такая фМРТ картина сопутствовала снижению аппетита и уменьшению калорийности потребляемой пищи. Исследование было выполнено на следующий день после проведенного курса лечения (рис. 5, 6).

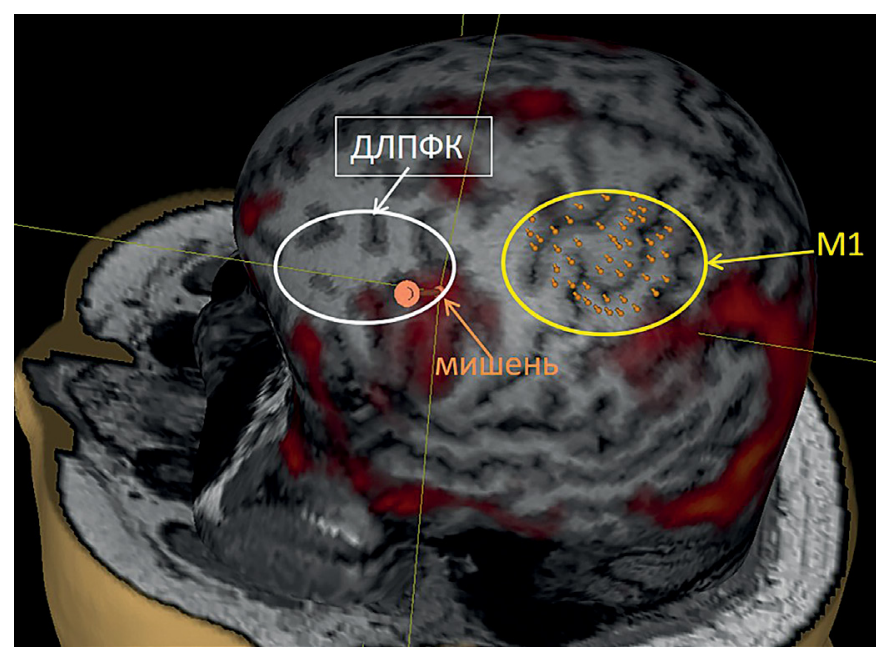

Рисунок 2. Изображение мишени для стимуляции, соответствующей активации в левой ДЛПФК в нейронавигационной системе (пациентка Ш.).

M1 - первичная моторная кора; ДЛПФК - дорсолатеральная префронтальная кора 


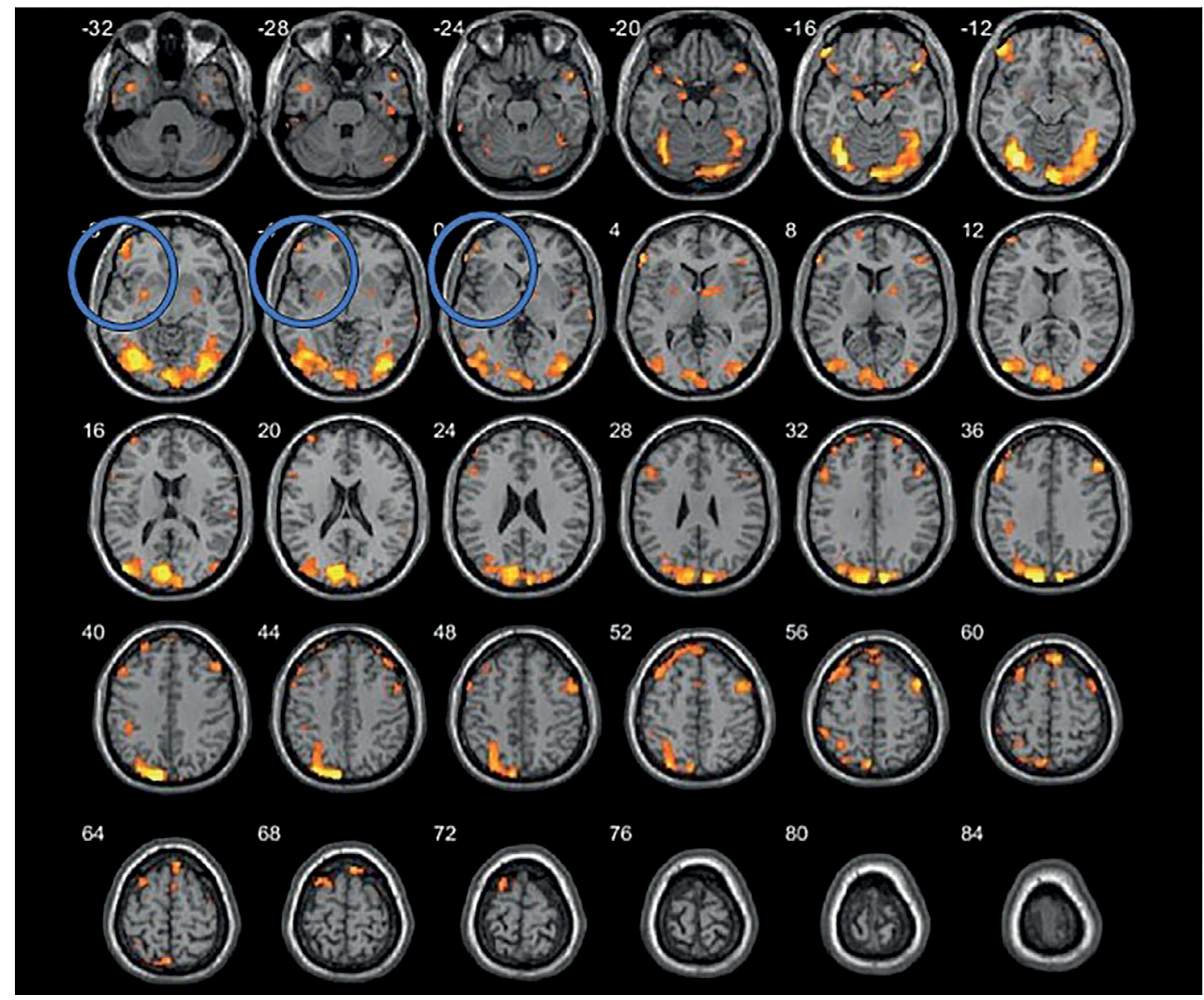

Рисунок 3. Данные функциональной МРТ (до лечения), синим кругом выделена область активации в зоне интереса (левая ДлПФК) (пациентка Ш.)

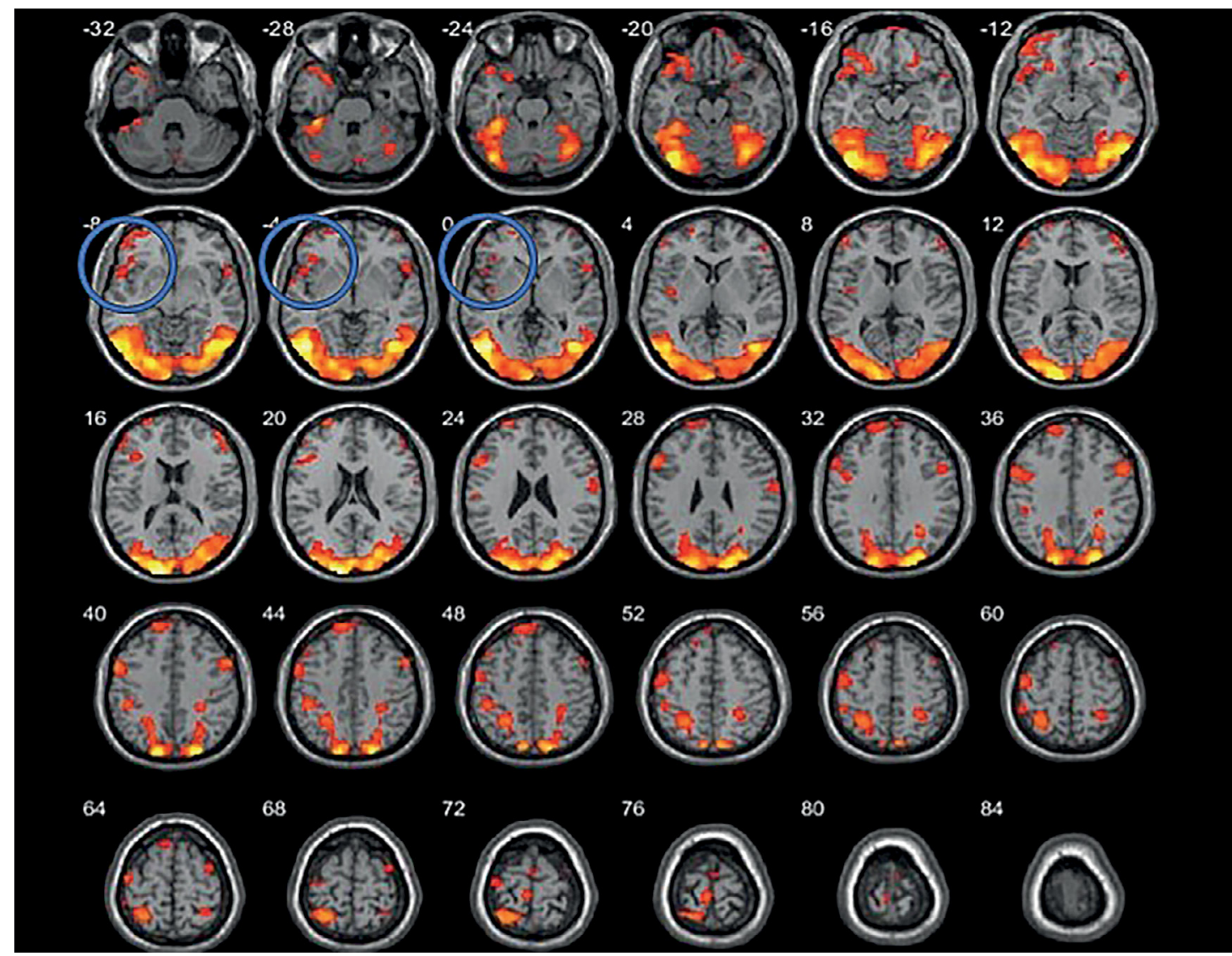

Рисунок 4. Данные функциональной МРТ (до лечения), синим выделена область активации в зоне интереса (левая ДлПФК) (пациентка Д.) 


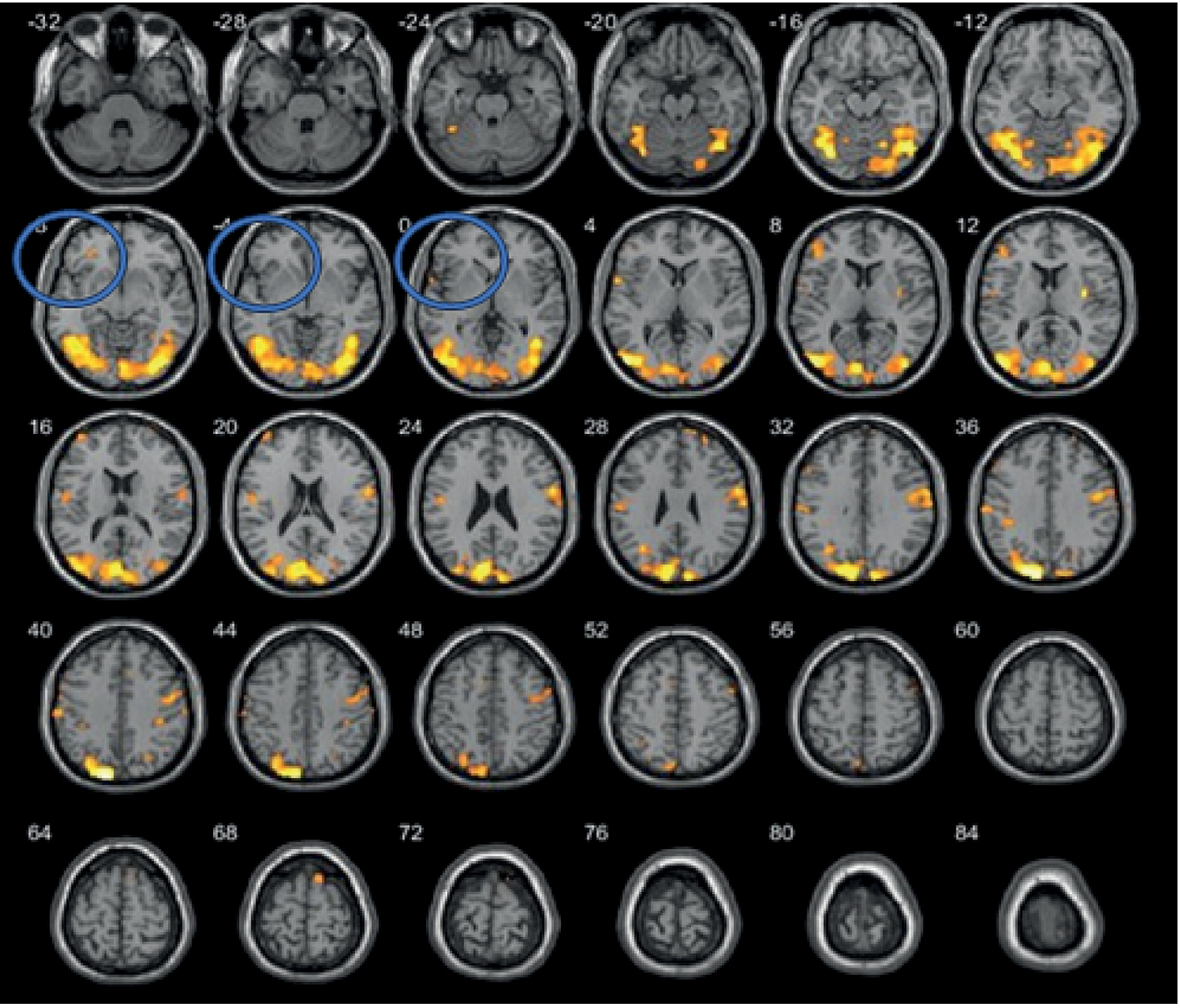

Рисунок 5. Данные функциональной МРТ (после лечения): отмечается отсутствие активации в зоне интереса (левая ДЛПФК) (пациентка Ш.)

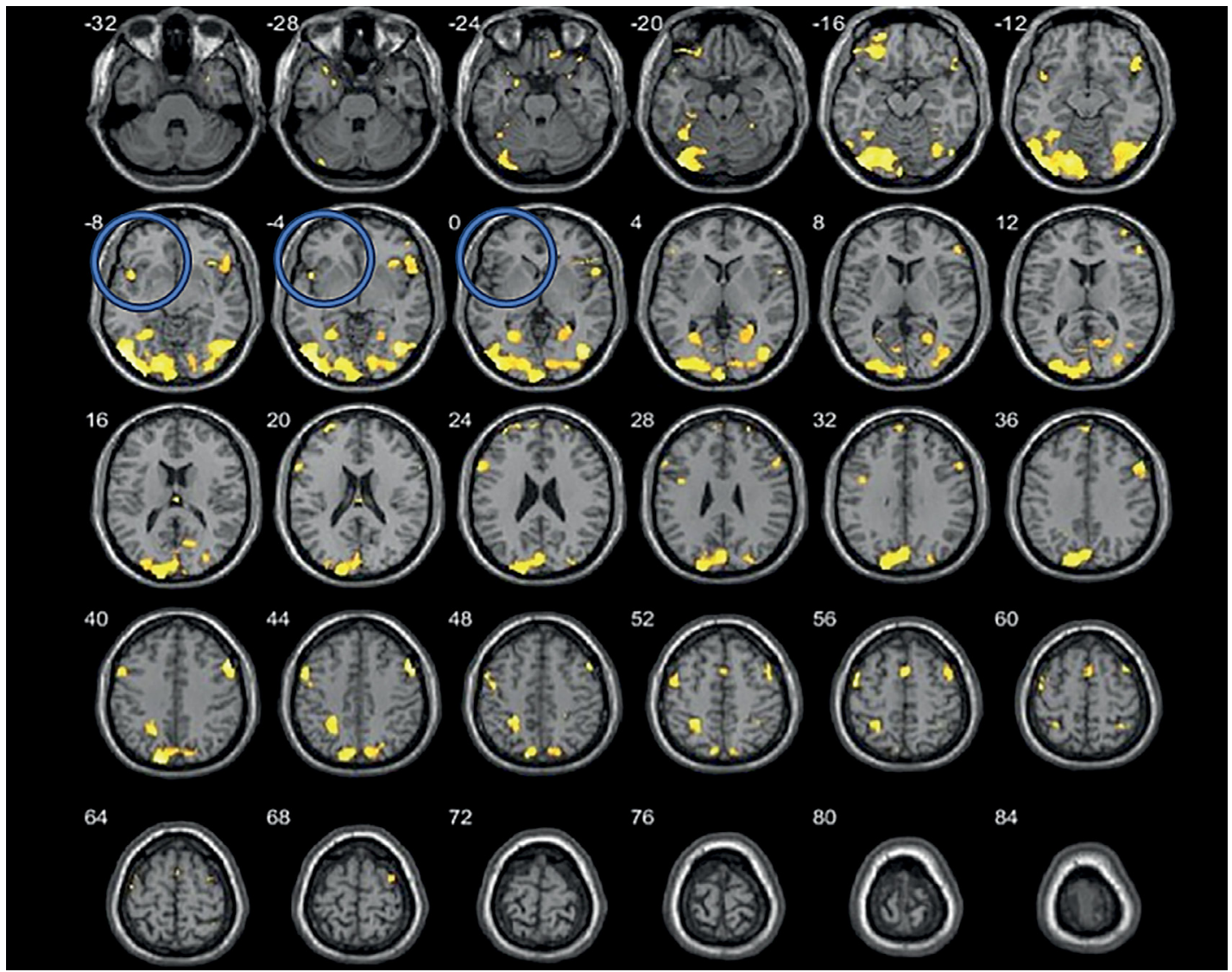

Рисунок 6. Данные функциональной МРТ (после лечения): отмечается отсутствие активации в зоне интереса (левая ДЛПФК) (пациентка Д.) 


\section{ОБСУЖДЕНИЕ}

Различные ассоциации эндокринологов во всем мире придерживаются позиции, что избыточная масса тела является хроническим заболеванием, и подчеркивают необходимость борьбы с этой глобальной эпидемией всеми возможными (но с доказанной безопасностью) методами, что обусловливает необходимость дальнейшего поиска для решения этой проблемы. С учетом важной регуляторной роли центральной нервной системы в формировании пищевого поведения, можно представить ожирение как нейроэндокринологическую проблему.

Механизмы влияния области ДЛПФК на пищевое поведение недостаточно хорошо изучены. В частности, изменение степени вознаграждения или расширения возможного эмоционально-волевого контроля за потреблением пищи рассматривается как одна из возможных регуляторных ролей ДЛПФК [39-41]. С функционированием именно этой зоны связывают и способность к сознательному ограничению себя в пристрастиях (в том числе, пищевых). Возможно, изменение активности в зоне ДЛПФК после сеансов рТМС способствует успешному подавлению тяги к пище за счет повышения когнитивного контроля. Несмотря на это, могут иметь место и другие причинно-следственные отношения, иным образом описывающие влияние рTMC на формирование пищевых парадигм.

В настоящей работе представлен небольшой, но перспективный опыт применения неинвазивной методики коррекции избыточного веса, основанный на комплекс- ном подходе. Данный положительный опыт может явиться началом более масштабных исследований в этой области.

\section{ЗАКЛЮЧЕНИЕ}

Представленные клинические случаи иллюстрируют тот факт, что применение метода навигационной рТМС представляется перспективным для коррекции пищевого поведения у пациентов с первичным ожирением. Однако требуется проведение дальнейших исследований с целью подбора оптимального протокола стимуляции, а также оценки ее долгосрочного эффекта.

\section{ДОПОЛНИТЕЛЬНАЯ ИНФОРМАЦИЯ}

Источник финансирования. Подготовка и публикация рукописи были выполнены в рамках темы Госзадания «Новые возможности диагностики и верификации патогенетических аспектов развития и прогрессирования цереброваскулярных заболеваний», НИОКТР №115013010109.

Согласие пациента. Пациенты добровольно подписали информированное согласие на публикацию персональной медицинской информации в обезличенной форме в журнале «Ожирение и метаболизм».

Конфликт интересов. Авторы декларируют отсутствие явных и потенциальных конфликтов интересов, связанных с публикацией настоящей статьи.

Участие авторов. Все авторы внесли значимый вклад в подготовку статьи, прочли и одобрили финальную версию статьи перед публикацией.

\section{СПИСОК ЛИТЕРАТУРЫ | REFERENCES}

1. Seidell JC, Halberstadt J. The global burden of obesity and the challenges of prevention. Ann Nutr Metab. 2015;66 Suppl 2:7-12. doi: https://doi.org/10.1159/000375143

2. Zentenius E, Andersson-Assarsson JC, Carlsson LMS, et al. Self-Reported Weight-Loss Methods and Weight Change: Ten-Year Analysis in the Swedish Obese Subjects Study Control Group. Obesity (Silver Spring). 2018;26(7):1137-1143. doi: https://doi.org/10.1002/oby.22200

3. Ralston J, Brinsden H, Buse $\mathrm{K}$, et al. Time for a new obesity narrative. Lancet. 2018;392(10156):1384-1386. doi: https://doi.org/10.1016/s0140-6736(18)32537-6

4. Логвинова О.В., Галиева М.О., Мазурина Н.В., Трошина Е.А Место препаратов центрального действия в алгоритмах лечения экзогенно-конституционального ожирения. // Ожирение и метаболизм. — 2017. — Т. 14. — №2 — С. 18-23. [Logvinova OV, Galieva MO, Mazurina NV, Troshina EA. The place of central-acting drugs in the algorithms of treatment of primary obesity. Obesity and metabolism. 2017;14(2):18-23. (In Russ.)] doi: https://doi.org/10.14341/OMET2017218-23

5. Bray GA, Kim KK, Wilding JPH, World Obesity F. Obesity: a chronic relapsing progressive disease process. A position statement of the World Obesity Federation. Obes Rev. 2017;18(7):715-723. doi: https://doi.org/10.1111/obr.12551

6. Schnabel RB, Yin X, Larson MG, et al. Multiple inflammatory biomarkers in relation to cardiovascular events and mortality in the community. Arterioscler Thromb Vasc Biol. 2013;33(7):1728-1733. doi: https://doi.org/10.1161/ATVBAHA.112.301174

7. Radmard AR, Merat S, Kooraki S, et al. Gallstone disease and obesity: a population-based study on abdominal fat distribution and gender differences. Ann Hepatol. 2015;14(5):702-709.

8. Giel KE, Hartmann A, Zeeck A, et al. Decreased Emotional Perception in Obesity. Eur Eat Disord Rev. 2016;24(4):341-346. doi: https://doi.org/10.1002/erv.2444

9. Вахмистров А.В. Клинико-психологический анализ различных форм эмоциогенного пищевого поведения. // Альманах клинической медицины. - 2001. — №4. - С. 127-130. [Vakhmistrov AV. Kliniko-psikhologicheskiy analiz razlichnykh form emotsiogennogo pishchevogo povedeniya. Almanac of clinical medicine. 2001;(4):127-130. (In Russ.)]

10. van Strien T, Frijters JER, Bergers GPA, Defares PB. The Dutch Eating Behavior Questionnaire (DEBQ) for assessment of restrained, emotional, and external eating behavior. Int J Eat Disord. 1986:5(2):295-315. doi: https://doi.org/10.1002/1098108x(198602)5:2<295::aid-eat2260050209>3.0.co;2-t

11. Baothman OA, Zamzami MA, Taher I, et al. The role of Gut Microbiota in the development of obesity and Diabetes. Lipids Health Dis. 2016;15(1). doi: https://doi.org/10.1186/s12944-016-0278-4

12. Kallus SJ, Brandt LJ. The intestinal microbiota and obesity. J Clin Gastroenterol. 2012;46(1):16-24. doi: https://doi.org/10.1097/MCG.0b013e31823711fd

13. Opinto G, Natalicchio A, Marchetti P. Physiology of incretins and loss of incretin effect in type 2 diabetes and obesity. Arch Physiol Biochem. 2013;119(4):170-178. doi: https://doi.org/10.3109/13813455.2013.812664

14. Apovian CM, Aronne LJ, Bessesen DH, et al. Pharmacological management of obesity: an endocrine Society clinical practice guideline. J Clin Endocrinol Metab. 2015;100(2):342-362. doi: https://doi.org/10.1210/jc.2014-3415

15. Yanovski SZ, Yanovski JA. Long-term Drug Treatment for Obesity. Jama. 2014;311(1):74. doi: https://doi.org/10.1001/jama.2013.281361

16. Greenway FL, Caruso MK. Safety of obesity drugs. Expert Opin Drug Saf. 2005;4(6):1083-1095. doi: https://doi.org/10.1517/14740338.4.6.1083

17. Pontiroli AE, Morabito A. Long-term prevention of mortality in morbid obesity through bariatric surgery. a systematic review and meta-analysis of trials performed with gastric banding and gastric bypass. Ann Surg. 2011;253(3):484-487. doi: https://doi.org/10.1097/SLA.0b013e31820d98cb 
18. Padwal R, Klarenbach S, Wiebe N, et al. Bariatric surgery: a systematic review and network meta-analysis of randomized trials. Obes Rev. 2011;12(8):602-621. doi: https://doi.org/10.1111/j.1467-789X.2011.00866.x

19. Wadden TA, Sarwer DB. Behavioral assessment of candidates for bariatric surgery: a patient-oriented approach. Surg Obes Relat Dis. 2006;2(2):171-179. doi: https://doi.org/10.1016/j.soard.2006.03.011

20. Lowe CJ, Vincent C, Hall PA. Effects of Noninvasive Brain Stimulation on Food Cravings and Consumption: A Meta-Analytic Review. Psychosom Med. 2017;79(1):2-13. doi: https://doi.org/10.1097/PSY.0000000000000368

21. Di Lazzaro V, Ziemann U, Lemon RN. State of the art: Physiology of transcranial motor cortex stimulation. Brain Stimul. 2008;1 (4):345-362. doi: https://doi.org/10.1016/j.brs.2008.07.004

22. Hoogendam JM, Ramakers GM, Di Lazzaro V. Physiology of repetitive transcranial magnetic stimulation of the human brain. Brain Stimul. 2010;3(2):95-118. doi: https://doi.org/10.1016/j.brs.2009.10.005

23. Chervyakov AV, Chernyavsky AY, Sinitsyn DO, Piradov MA. Possible Mechanisms Underlying the Therapeutic Effects of Transcranial Magnetic Stimulation. Front Hum Neurosci. 2015;9:303. doi: https://doi.org/10.3389/fnhum.2015.00303

24. Червяков А.В., Пойдашева А.Г., Коржова Ю.Е., и др. Ритмическая транскраниальная магнитная стимуляция в неврологии и психиатрии. // Журнал неврологии и психиатрии им. С.С. Корсакова. 2015. - Т. 115. — №12. - С. 7-18. [Chervyakov AV, Poydasheva AG, Korzhova JE, et al. Repetitive transcranial magnetic stimulation in neurology and psychiatry. Zh Nevrol Psikhiatr Im S S Korsakova. 2015;115(12):7-18. (In Russ.)] doi: https://doi.org/10.17116/jnevro20151151127-18

25. Bliss TV, Cooke SF. Long-term potentiation and long-term depression: a clinical perspective. Clinics (Sao Paulo). 2011;66 Suppl 1:3-17 doi: https://doi.org/10.1590/s1807-59322011001300002

26. Duffau H. Brain plasticity: from pathophysiological mechanisms to therapeutic applications. J Clin Neurosci. 2006;13(9):885-897. doi: https://doi.org/10.1016/j.jocn.2005.11.045

27. Val-Laillet D, Aarts E, Weber B, et al. Neuroimaging and neuromodulation approaches to study eating behavior and prevent and treat eating disorders and obesity. Neuroimage Clin. 2015;8:1-31 doi: https://doi.org/10.1016/j.nicl.2015.03.016

28. Hall PA, Vincent CM, Burhan AM. Non-invasive brain stimulation for food cravings, consumption, and disorders of eating: A review of methods, findings and controversies. Appetite. 2018;124:78-88. doi: https://doi.org/10.1016/j.appet.2017.03.006

29. Uher R, Yoganathan D, Mogg A, et al. Effect of left prefrontal repetitive transcranial magnetic stimulation on food craving. Biol Psychiatry. 2005:58(10):840-842 doi: https://doi.org/10.1016/j.biopsych.2005.05.043

30. Van den Eynde F, Claudino AM, Mogg A, et al. Repetitive transcranial magnetic stimulation reduces cue-induced food craving in bulimic disorders. Biol Psychiatry. 2010;67(8):793-795 doi: https://doi.org/10.1016/j.biopsych.2009.11.023
31. Kim SH, Chung JH, Kim TH, et al. The effects of repetitive transcranial magnetic stimulation on eating behaviors and body weight in obesity: A randomized controlled study. Brain Stimul. 2018;11(3):528-535. doi: https://doi.org/10.1016/j.brs.2017.11.020

32. Логвинова О.В., Пойдашева А.Г., Бакулин И.С., и др. Современные представления о патогенезе ожирения и новых подходах к его коррекции. // Ожирение и метаболизм. - 2018. - Т. 15. №2. - C. 11-16. [Logvinova OV, Poydasheva AG, Bakulin IS, et al. Modern concepts of the pathogenesis of obesity and new approaches to its correction. Obesity and metabolism. 2018;15(2):11-16. (In Russ.)] doi: https://doi.org/10.14341/OMET9491

33. Heinitz S, Reinhardt M, Piaggi P, et al. Neuromodulation directed at the prefrontal cortex of subjects with obesity reduces snack food intake and hunger in a randomized trial. Am J Clin Nutr. 2017;106(6):1347-1357. doi: https://doi.org/10.3945/ajcn.117.158089

34. Ruohonen J, Karhu J. Navigated transcranial magnetic stimulation. Neurophysio/ Clin. 2010;40(1):7-17. doi: https://doi.org/10.1016/j.neucli.2010.01.006

35. Ahdab R, Ayache SS, Brugieres P, et al. Comparison of «standard» and «navigated» procedures of TMS coil positioning over motor, premotor and prefrontal targets in patients with chronic pain and depression. Neurophysiol Clin. 2010;40(1):27-36. doi: https://doi.org/10.1016/j.neucli.2010.01.001

36. Willis LH, Slentz CA, Bateman LA, et al. Effects of aerobic and/or resistance training on body mass and fat mass in overweight or obese adults. J Appl Physiol (1985). 2012;113(12):1831-1837. doi: https://doi.org/10.1152/japplphysiol.01370.2011

37. Poirier P, Després J-P. Exercise in Weight Management of Obesity. Cardiol Clin. 2001;19(3):459-470. doi: https://doi.org/10.1016/s0733-8651(05)70229-0

38. Rossi S, Hallett M, Rossini PM, et al. Safety, ethical considerations, and application guidelines for the use of transcranial magnetic stimulation in clinical practice and research. Clin Neurophysiol. 2009;120(12):2008-2039. doi: https://doi.org/10.1016/j.clinph.2009.08.016

39. Gluck ME, Viswanath P, Stinson EJ. Obesity, Appetite, and the Prefrontal Cortex. Curr Obes Rep. 2017;6(4):380-388. doi: https://doi.org/10.1007/s13679-017-0289-0

40. Keller KL. Brain stimulation for treatment of obesity: will stimulating the prefrontal cortex reduce overeating? Am J Clin Nutr. 2017;106(6):1331-1332. doi: https://doi.org/10.3945/ajcn.117.169631

41. Кремнева Е.И., Суслин А.С., Говорин А.Н., и др. ФМРТкартирование алиментарных функциональных зон головного мозга. // Анналы клинической и экспериментальной неврологии. - 2015. - T. 9. - №1. - C. 32-36. [Kremneva El, Suslin AS, Govorin AN, et al. Mapping of the brain regions responsible for eating behavior regulation with functional MRI. Annaly klinicheskoy i eksperimental'noy nevrologii. 2015;9(1):32-36. (In Russ.)]

\section{ИНФОРМАЦИЯ ОБ АВТОРАХ [AUTHORS INFO]:}

*Кузнецова Полина Игоревна, к.м.н. [Polina I. Kuznetsova, MD, PhD]; адрес: Россия, 125367, Москва, Волоколамское шоссе, д. 80 [address: 80 Volokolamskoe shosse, 1125367 Moscow, Russia]; ORCID: http://orcid.org/0000-0002-4626-6520; eLibrary SPIN: 5105-5173; e-mail: angioneurology0@gmail.com

Логвинова Оксана Викторовна, аспирант [Oksana V. Logvinova, MD, postgraduate student]; ORCID: http://orcid.org/0000-0002-6862-7323; eLibrary SPIN: 7376-3911; e-mail: dr.logvinova@yandex.ru Пойдашева Александра Георгиевна [Alexandra G. Poydasheva, MD]; ORCID: http://orcid.org/0000-0003-1841-1177; eLibrary SPIN: 4040-1184 ; e-mail: alexandra.poydasheva@gmail.com

Кремнева Елена Игоревна, к.м.н. [Elena I. Kremneva, MD, PhD]; ORCID: http://orcid.org/0000-0001-9396-6063; eLibrary SPIN: 8799-8092; e-mail: kremneva@neurology.ru

Бакулин Илья Сергеевич, к.M.H. [llya S. Bakulin, MD, PhD]; ORCID: http://orcid.org/0000-0003-0716-3737; eLibrary SPIN: 7756-6427; e-mail: bakulinilya@gmail.com

Раскуражев Антон Алексеевич, к.M.H. [Anton A. Raskurazhev, MD, PhD]; ORCID: http://orcid.org/0000-0003-0522-767X; eLibrary SPIN: 9207-5195; e-mail: rasckey@live.com

Лагода Ольга Викторовна, к.м.н. [Olga V. Lagoda, MD, PhD]; ORCID: http://orcid.org/0000-0001-7562-4991; eLibrary SPIN: 1789-4735; e-mail: olga.lagoda@gmail.com

Супонева Наталья Александровна, д.м.н. [Natalia A. Suponeva, MD, PhD];

ORCID: http://orcid.org/0000-0003-3956-6362; eLibrary SPIN: 3223-6006; e-mail: nasu2709@mail.ru 
Трошина Екатерина Анатольевна, д.м.н., професcop [Ekaterina A. Troshina, MD, PhD, professor]; ORCID: http://orcid.org/0000-0002-8520-8702; eLibrary SPIN: 8821-8990; e-mail: troshina@inbox.ru

Танашян Маринэ Мовсесовна, Д.М.н., профессор [Marine M. Tanashyan, MD, PhD, professor];

ORCID: http://orcid.org/0000-0002-5883-8119; eLibrary SPIN: 7191-1163; e-mail: mtanashyan@neurology.ru

Пирадов Михаил Александрович, д.м.н., професcop [Michael A. Piradov, MD, PhD, professor]; ORCID: http://orcid.org/0000-0002-6338-0392; eLibrary SPIN: 2860-1689; e-mail: mpi711@gmail.com

*Автор, ответственный за переписку / Corresponding author.

\section{ЦИТИРОВАТЬ:}

Кузнецова П.И., Логвинова О.В., Пойдашева А.Г., Кремнева Е.И., Бакулин И.С., Раскуражев А.А., Лагода О.В., Супонева Н.А., Трошина Е.А., Танашян М.М., Пирадов М.А. Применение навигационной ритмической транскраниальной магнитной стимуляции с целью коррекции пищевого поведения при ожирении (клинические наблюдения) // Ожирение и метаболизм. - 2020. - Т. 17. - №1. - С. 100-109. doi: https://doi.org/10.14341/omet10148

\section{TO CITE THIS ARTICLE:}

Kuznetsova PI, Logvinova OV, Poydasheva AG, Kremneva El, Bakulin IS, Raskurazhev AA, Lagoda OV, Suponeva NA, Troshina EA, Tanashyan MM, Piradov MA. Navigated repetitive transcranial magnetic stimulation to correct eating behavior in obesity (clinical cases). Obesity and metabolism. 2020;17(1):100-109. doi: https://doi.org/10.14341/omet10148 